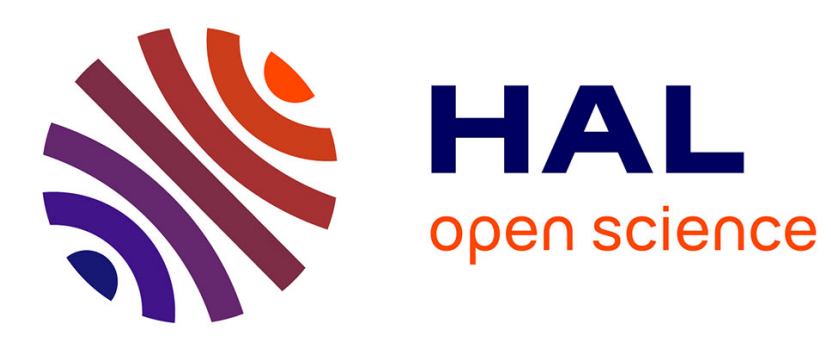

\title{
Orientational Effect of the In-Plane Magnetic Field on the FFLO Modulation in Layered Superconductors
}

\author{
Mihail D. Croitoru, Alexandre I. Buzdin
}

\section{To cite this version:}

Mihail D. Croitoru, Alexandre I. Buzdin. Orientational Effect of the In-Plane Magnetic Field on the FFLO Modulation in Layered Superconductors. Journal of Superconductivity and Novel Magnetism, 2013, 26 (5), pp.1657-1661. 10.1007/s10948-012-2083-2 . hal-00820842

\section{HAL Id: hal-00820842 \\ https://hal.science/hal-00820842}

Submitted on 1 Mar 2018

HAL is a multi-disciplinary open access archive for the deposit and dissemination of scientific research documents, whether they are published or not. The documents may come from teaching and research institutions in France or abroad, or from public or private research centers.
L'archive ouverte pluridisciplinaire HAL, est destinée au dépôt et à la diffusion de documents scientifiques de niveau recherche, publiés ou non, émanant des établissements d'enseignement et de recherche français ou étrangers, des laboratoires publics ou privés.

\section{(ㄷ)(1) $\$$}

Distributed under a Creative Commons Attribution - NonCommerciall 4.0 International 


\title{
Orientational Effect of the In-Plane Magnetic Field on the FFLO Modulation in Layered Superconductors
}

\author{
M.D. Croitoru • A.I. Buzdin
}

\begin{abstract}
We present the analysis of the Fulde-FerrellLarkin-Ovchinnikov phase in an s-wave superconducting layered system in parallel magnetic field. We show that in the close vicinity of the tricritical point in the system with elliptical Fermi surface the orbital effect pins the FFLO modulation vector perpendicular to the applied magnetic field. At low temperatures, the FFLO modulation can acquire different directions.
\end{abstract}

Almost five decades ago, Larkin and Ovchinnikov [1], at the
same time as Fulde and Ferrell [2], suggested that in clean
superconductors at $T<0.56 T_{c 0}$ a paired state described by
the spatially modulated order parameter becomes favorable
when the spin effect of the magnetic field dominates over
the orbital effect of the magnetic field. Usually, it is an or-
bital effect, which is a most important and this made diff-
cult the experimental observation of the FFLO phase. More-
over, the superconductor must be in the clean limit because
electron scattering is detrimental for the FFLO phase [3].
The orbital effect may be weakened in heavy fermion super-
conductors or in quasi-2D superconductors when external
magnetic field is aligned along the conducting planes. In-
deed, there have been observed several hints indicating the
experimental realization of the FFLO state in organic super-
conductors and in heavy fermion superconductors [4-16].
In particular, the anomaly in the thermal conductivity for

M.D. Croitoru ( $\varangle)$ · A.I. Buzdin

Université Bordeaux I LOMA, UMR 5798, 33400 Talence,

France

e-mail: mikhail.croitoru@uni-bayreuth.de

\section{A.I. Buzdin}

Institut Universitaire de France, Paris, France the clean organic sample $\lambda$-(BETS $)_{2} \mathrm{GaCl}_{4}$, [5] the calorimetric and magnetic torque evidence for the appearance of an additional first-order phase transition line within the superconducting phase in the in-plane high field regime for organic sample $\kappa$-(BEDT-TTF $)_{2} \mathrm{Cu}(\mathrm{NCS})_{2}[10,13]$, as well as an anomalous in-plane anisotropy of the onset of superconductivity in (TMTSF) ${ }_{2} \mathrm{ClO}_{4}$ conductor [17] have been interpreted as related to a stabilization of the superconducting phase with the modulated order parameter in real space. A second specific heat anomaly within the superconducting state evidences the signature of the FFLO modulated superconducting phase in the high-field low-temperature regime in the heavy-fermion compound $\mathrm{CeCoIn}_{5}[6,7]$.

In $[18,19,21]$, we have demonstrated that the FFLO modulation strongly interferes with the orbital effect and provides the main source of the in-plane critical field anisotropy. Our subsequent analysis of the properties of the organic superconductors, which are the candidates to the FFLO phase realization, revealed that in some of them the resonance between the period of the FFLO modulation and the period of the interlayer coupling modulation induced by the external field is possible [20]. The obtained results predict the anomalous cusps in the temperature and angular dependencies of the in-plane critical field. Their experimental observation may serve as an unambiguous evidence for the appearance of the FFLO phase in layered superconductors. In these studies, we assumed that small deviations of the Fermi surface from elliptical form pins the direction of the FFLO vector. In reality, the FFLO vector can be changed by orbital effects, also. Therefore, in this work, we investigate the opposite situation, when deviation of the Fermi surface from the ellipticity is negligible and find the direction of the vector $\mathbf{q}$. 


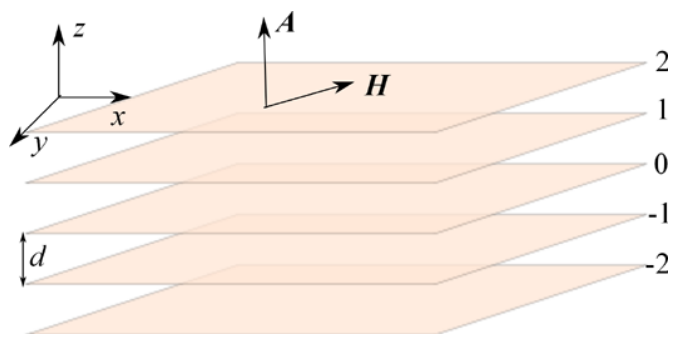

Fig. 1 Scheme of the quasi-2D layered metal

The single-electron spectrum is approximated by

$E_{\mathbf{p}}=\frac{p_{x}^{2}}{2 m_{x}}+\frac{p_{y}^{2}}{2 m_{y}}+2 t \cos \left(p_{z} d\right)$

where $\mathbf{p}=\left(p_{x}, p_{y}, p_{z}\right)$ is the electron momentum. The corrugation of the Fermi surface due to the coupling between adjacent layers (interlayer distance $d$, see Figs. 1 and 2) is assumed to be small, i.e., $t \ll T_{c 0}$, but sufficiently large to make the mean-field treatment valid, $\left|\ln \left(T_{c 0} / t\right)\right| T_{c 0} /$ $E_{F} \ll 1$ [22]. Here, $T_{c 0}$ is the critical temperature of the system at zero magnetic field and $E_{F}$ is the Fermi energy. We choose a gauge for which the vector potential $\mathbf{A}=\mathbf{H} \times \mathbf{r}[\mathbf{r}=(x, y, 0)$ is a coordinate in $x y$-plane], i.e., $A_{z}=-x H \sin \alpha+y H \cos \alpha$, where $\alpha$ is the angle between the applied field, with amplitude $H$, and $x$-axis. As was demonstrated in $[22,23]$, the anisotropic model with effective masses can be reduced to the isotropic one by a scaling transformation and corresponding renormalization of the magnetic field. Performing this scaling transformation, we will thus consider from now on an isotropic in-plane spectrum, with mass $m=m_{x}$, and a magnetic field $\mathbf{H}=$ $H\left[\left(m_{x} / m_{y}\right)^{1 / 2} \cos \alpha, \sin \alpha, 0\right]$. Taking into account that the system is near the second-order phase transition, the linearized Eilenberger equation on the anomalous Green function $f_{\omega}\left(\mathbf{n}, \mathbf{r}, p_{z}\right)$ describing layered superconducting systems acquires the form (for positive Matsubara frequency $\omega$ at temperature $T)$ [25]

$$
\begin{aligned}
& {\left[\omega+i h+\frac{1}{2} \mathbf{v}_{F} \cdot \nabla+2 i t \sin \left(p_{z} d\right) \sin (\mathbf{Q} . \mathbf{r})\right] f_{\omega}\left(\mathbf{n}, \mathbf{r}, p_{z}\right)} \\
& \quad=\Delta(\mathbf{r}) .
\end{aligned}
$$

Here, $h=\mu_{B} H$ is the Zeeman energy, $\mathbf{v}_{F}=v_{F} \mathbf{n}$ is the in-plane Fermi velocity, and $\mathbf{Q}=\left(\pi d H / \phi_{0}\right)[-\sin \alpha$, $\left.\left(m_{x} / m_{y}\right)^{1 / 2} \cos \alpha, 0\right]$ with $\phi_{0}=\pi c / e$. The order parameter is defined self-consistently as

$\frac{1}{\lambda} \Delta(\mathbf{r})=2 \pi T \Re \sum_{\omega>0}\left\langle f_{\omega}\left(\mathbf{n}, \mathbf{r}, p_{z}\right)\right\rangle$,

where $\lambda$ is the BCS pairing constant and the brackets denote averaging over $p_{z}$ and $\mathbf{n}$. Here, we considered a layered superconductor in the clean limit, meaning that the in-plane

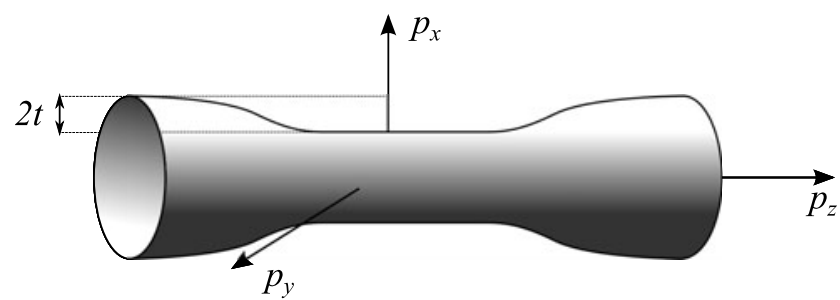

Fig. 2 The Fermi surface of the layered metal in the form of a corrugated cylinder

mean free path is much larger than the corresponding coherence length, $\xi_{0}=v_{F} /\left(2 \pi T_{c 0}\right)$. The upper critical field corresponds to the values of $H$ for which the system of Eqs. (2) and (3) can be solved.

The solution of the Eilenberger equation (2) can be chosen without loss of generality as

$f_{\omega}\left(\mathbf{n}, \mathbf{r}, p_{z}\right)=e^{i \mathbf{q r}} \sum_{m} e^{i m \mathbf{Q} \cdot \mathbf{r}} f_{m}\left(\omega, \mathbf{n}, p_{z}\right)$.

Equation (4) takes into account the possibility for the formation of the pairing state $\left(\mathbf{k}+\frac{\mathbf{q}}{2}, \uparrow ;-\mathbf{k}+\frac{\mathbf{q}}{2}, \downarrow\right)$ with finite center-of-mass momentum. At the same time, the order parameter can be expanded as

$\Delta(\mathbf{r})=e^{i \mathbf{q r}} \sum_{m} e^{i 2 m \mathbf{Q} \cdot \mathbf{r}} \Delta_{2 m}$.

Retaining terms up to the second order in $t / T_{c 0}$, we obtain

$$
\begin{aligned}
\ln \frac{T_{c 0}}{T_{c}}= & 2 \pi T_{c} \Re \sum_{\omega>0}\left\{\frac{1}{\omega}-\frac{1}{L(\mathbf{q})}\right) \\
& \left.\left.+\frac{\tilde{t}^{2}}{L^{2}(\mathbf{q})}\left(\frac{1}{L(\mathbf{q}+\mathbf{Q})}+\frac{1}{L(\mathbf{q}-\mathbf{Q})}\right)\right)\right\},
\end{aligned}
$$

where $L_{n}(\mathbf{x})=\omega_{n}+i h+i \mathbf{v}_{F} \mathbf{x} / 2$. This equation defines the magnetic field dependence of the superconducting onset temperature $T_{c}$ in layered superconductors, when both the paramagnetic and orbital effects are accounted for. The magnitude of the FFLO modulation vector $\mathbf{q}$ is determined by the condition of the maximum value of the upper critical field in the Pauli limited regime via equation

$\left.\ln \left(\frac{T_{c 0}}{T_{c P}}\right)=\pi T_{c P} \sum_{n} \frac{1}{\omega}-\frac{1}{L(\mathbf{q})}\right\rangle$,

where we introduced $T_{c P}$, the corresponding superconducting onset temperature.

In the close vicinity of the tricritical point (TCP, $T^{*} \simeq$ $0.56 T_{c 0}$ or $\left.H^{*} \simeq 1.06 T_{c 0} / \mu_{B}\right)$, the contribution of the orbital part can be found analytically. Expanding Eq. (6) up to the fourth order in $\mathbf{q}$ and $\mathbf{Q}$ and performing summation 
over $\omega_{n}$, one obtains the following equation:

$$
\begin{aligned}
\ln \frac{T_{c 0}}{T_{c}}= & \ln (4 \gamma)+\psi^{(0)}\left(\frac{1}{2}+\frac{i h}{2 \pi T}\right) \\
& -\frac{1}{8(2 \pi T)^{2}}\left[\left\langle\left(\mathbf{v}_{F} \mathbf{q}\right)^{2}\right\rangle+4 t^{2}\right] \psi^{(2)}\left(\frac{1}{2}+\frac{i h}{2 \pi T}\right) \\
& +\frac{1}{384(2 \pi T)^{4}}\left[\left\langle\left(\mathbf{v}_{F} \mathbf{q}\right)^{4}\right\rangle+24 t^{2}\left\langle\left(\mathbf{v}_{F} \mathbf{q}\right)^{2}\right\rangle\right. \\
& \left.+4 t^{2}\left\langle\left(\mathbf{v}_{F} \mathbf{Q}\right)^{2}\right\rangle\right] \psi^{(4)}\left(\frac{1}{2}+\frac{i h}{2 \pi T}\right) \\
& -\frac{t^{2}}{11520(2 \pi T)^{6}}\left[15\left\langle\left(\mathbf{v}_{F} \mathbf{q}\right)^{4}\right\rangle\right. \\
& +15\left\langle\left(\mathbf{v}_{F} \mathbf{q}\right)^{2}\left(\mathbf{v}_{F} \mathbf{Q}\right)^{2}\right\rangle \\
& \left.+\left\langle\left(\mathbf{v}_{F} \mathbf{Q}\right)^{4}\right\rangle\right] \psi^{(6)}\left(\frac{1}{2}+\frac{i h}{2 \pi T}\right) .
\end{aligned}
$$

This can be written as

$$
\begin{aligned}
\ln \frac{T_{c 0}}{T_{c}}= & \ln (4 \gamma)+\psi^{(0)}\left(\frac{1}{2}+\frac{i h}{2 \pi T}\right)-\frac{q^{2}}{8(2 \pi T)^{2}} \\
& \times\left[\left\langle v_{F x}^{2}\right\rangle \cos ^{2}(\phi)+\left\langle v_{F y}^{2}\right\rangle \sin ^{2}(\phi)+4 \frac{t^{2}}{q^{2}}\right] \\
& \times \psi \psi^{(2)}\left(\frac{1}{2}+\frac{i h}{2 \pi T}\right)+\frac{q^{4}}{384(2 \pi T)^{4}} \\
& \times\left\{\left\langle v_{F x}^{4}\right\rangle \cos ^{4}(\phi)+6\left\langle v_{F x}^{2} v_{F y}^{2}\right\rangle \cos ^{2}(\phi) \sin ^{2}(\phi)\right. \\
& +\left\langle v_{F y}^{4}\right\rangle \sin ^{4}(\phi) \\
& +24 \frac{t^{2}}{q^{2}}\left[\left\langle v_{F x}^{2}\right\rangle \cos ^{2}(\phi)+\left\langle v_{F y}^{2}\right\rangle \sin ^{2}(\phi)\right] \\
& \left.+4 \frac{t^{2}}{q^{4}} K^{2}\left[\left\langle v_{F x}^{2}\right\rangle \sin ^{2}(\vartheta)+\left\langle v_{F y}^{2}\right\rangle \cos ^{2}(\vartheta)\right]\right\} \\
& \times \psi(4)\left(\frac{1}{2}+\frac{i h}{2 \pi T}\right)-\frac{q^{4} t^{2}}{768(2 \pi T)^{6}} \\
& \times\left\{\left\langle v_{F x}^{4}\right\rangle \cos ^{4}(\phi)+6\left\langle v_{F x}^{2} v_{F y}^{2}\right\rangle \cos ^{2}(\phi) \sin ^{2}(\phi)\right. \\
& +\left\langle v_{F y}^{4}\right\rangle \sin ^{4}(\phi)+\frac{K^{2}}{q^{2}}\left[\left\langle v_{F x}^{4}\right\rangle \cos ^{2}(\phi) \sin ^{2}(\vartheta)\right. \\
& +\left\langle v_{F y}^{4}\right\rangle \sin ^{2}(\phi) \cos ^{2}(\vartheta) \\
& +\left\langle v_{F x}^{2} v_{F y}^{2}\right\rangle\left[\sin ^{2}(\phi) \sin ^{2}(\vartheta)+\cos ^{2}(\phi) \cos ^{2}(\vartheta)\right. \\
& \left.\left.-4 \cos ^{4}(\phi) \sin _{F x}^{4}\right\rangle\right) \cos ^{4}(\vartheta)+6\left\langle v_{F x}^{2} v_{F y}^{2}\right\rangle \\
& \left.\left.\sin ^{2}(\vartheta)\right]\right] \\
& \\
&
\end{aligned}
$$

$$
\begin{aligned}
& \left.\left.\times \sin ^{2}(\vartheta) \cos ^{2}(\vartheta)+\left\langle v_{F y}^{4}\right\rangle \cos ^{4}(\vartheta)\right]\right\} \\
& \times \psi^{(6)}\left(\frac{1}{2}+\frac{i h}{2 \pi T}\right) .
\end{aligned}
$$

where $K^{2}=\pi^{2} d^{2} H^{2} / \phi_{0}^{2}$, and $\psi(z)=\sum_{n=0}^{\infty}(n+z)^{-1}$.

In the case of elliptical Fermi surface, we can calculate $\left\langle v_{F x}^{4}\right\rangle=\left\langle v_{F y}^{4}\right\rangle=v_{F}^{4} \int_{0}^{2 \pi} \cos ^{4}(\varphi) \frac{d \varphi}{2 \pi}=\frac{3}{8} v_{F}^{4}$, and $\left\langle v_{F x}^{2} v_{F y}^{2}\right\rangle=\frac{1}{8} v_{F}^{4}$, where $\varphi$ is the angle between the $x$ axis and the direction of the $\mathbf{v}_{F}$ vector. Then $\left\langle\left(\mathbf{v}_{F} \mathbf{Q}\right)^{2}\right\rangle=$ $\frac{1}{2} K^{2} v_{F}^{2}, \quad\left\langle\left(\mathbf{v}_{F} \mathbf{Q}\right)^{4}\right\rangle=\frac{3}{8} K^{4} v_{F}^{4}, \quad\left\langle\left(\mathbf{v}_{F} \mathbf{q}\right)^{2}\right\rangle=\frac{1}{2} q^{2} v_{F}^{2}$ and $\left\langle\left(\mathbf{v}_{F} \mathbf{q}\right)^{4}\right\rangle=\frac{3}{8} v_{F}^{4} q^{4}$, and finally

$$
\left\langle\left(\mathbf{v}_{F} \mathbf{q}\right)^{2}\left(\mathbf{v}_{F} \mathbf{Q}\right)^{2}\right\rangle=\frac{1}{8} K^{2} q^{2} v_{F}^{4}[2-\cos (2 \phi-2 \vartheta)] .
$$

Therefore,

$$
\begin{aligned}
\ln \frac{T_{c 0}}{T_{c}}= & \ln (4 \gamma)+\psi^{(0)}\left(\frac{1}{2}+\frac{i h}{2 \pi T}\right) \\
& -\frac{1}{16(2 \pi T)^{2}}\left[q^{2} v_{F}^{2}+8 t^{2}\right] \psi^{(2)}\left(\frac{1}{2}+\frac{i h}{2 \pi T}\right) \\
& +\frac{v_{F}^{4}}{1024(2 \pi T)^{4}}\left[q^{4}+32 t^{2} \frac{q^{2}}{v_{F}^{2}}+16 t^{2} \frac{K^{2}}{3 v_{F}^{2}}\right] \\
& \times \psi^{(4)}\left(\frac{1}{2}+\frac{i h}{2 \pi T}\right)-\frac{t^{2} v_{F}^{4}}{6144(2 \pi T)^{6}} \\
& \times\left[3 q^{4}+K^{2} q^{2}[2-\cos (2 \phi-2 \vartheta)]+\frac{1}{5} K^{4}\right] \\
& \times \psi^{(6)}\left(\frac{1}{2}+\frac{i h}{2 \pi T}\right) .
\end{aligned}
$$

We see that the largest onset temperature is for $\mathbf{q} \| \mathbf{Q}$, i.e., the FFLO modulation vector is directed perpendicular to the external field $\mathbf{H}$. Thus, for the $s$-wave pairing and for the isotropic (elliptical) Fermi surface the magnetic field provides an orientational effect.

To make a definitive conclusion about the direction of the modulation, when external magnetic field is beyond the TCP region, we have to perform the summation over the Matsubara frequencies in Eq. (6) numerically. We used $N=10^{4}$ terms in the summation and this number suffices for convergency at $T / T_{c 0}>10^{-2}$. The calculations were performed for different values of the dimensionless Fermi velocity parameter $\eta=\hbar v_{F} \pi d / \phi_{0} \mu_{B} \quad(\eta=2.76$ corresponds to the Fermi velocity $v_{F}=1.0 \times 10^{7} \mathrm{~cm} / \mathrm{sec}$ and the interlayer distance $d=1.31 \mathrm{~nm}$ ).

Figure 3 shows the magnetic field angular dependence of the normalized superconducting transition temperature, $T_{c}(\alpha) / T_{c P}$, at constant modulus of the in-plane magnetic field and $t / T_{c 0}=0.2$. In the polar plot, the direction of each point seen from the origin corresponds to the magnetic-field 

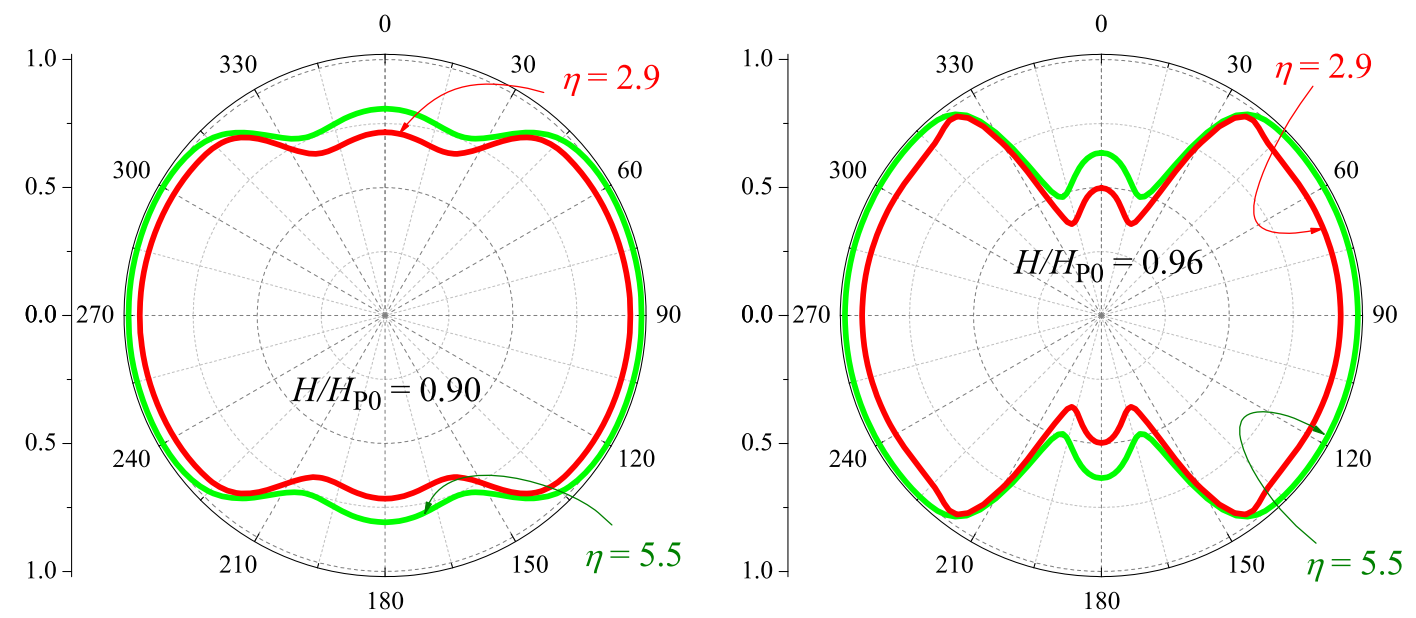

Fig. 3 The in-plane field-direction dependence of $T_{c} / T_{c P}$ at $T_{c P} / T_{c 0}=0.9$ (upper panel) and $T_{c P} / T_{c 0}=0.96$ (lower panel) for $\eta=2.9$ and $\eta=5.5$

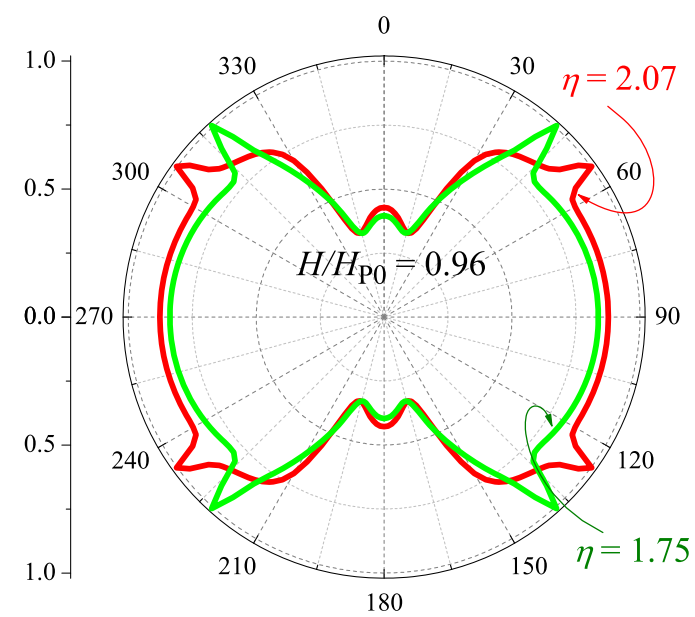

Fig. 4 The in-plane field-direction dependence of $T_{c} / T_{c P}$ at $T_{c P} / T_{c 0}=0.96$ for $\eta=1.76$ and $\eta=2.09$

direction and the distance from the origin corresponds to the normalized critical temperature, when the orbital destructive effect is taken into account. For a magnetic field below $H / H_{P 0} \simeq 0.9$ that corresponds to $T_{c P} / T_{c 0} \simeq 0.10$, the largest upper critical field is for angle $\alpha= \pm 90^{\circ}$ that magnetic field makes from the $x$-axis. So, we get the same result $\mathbf{q} \perp \mathbf{H}$. Here, $H_{P 0}=\Delta_{0} / \mu_{B}$ is the critical magnetic field at $T=0$ in Pauli limited two-dimensional superconductors [26]. For higher magnetic fields, there can develop a situation when the FFLO modulation vector is tilted, as shown in the lower panel of Fig. 3 for $\eta=2.9$. In this particular case, the angle between the $\mathbf{q}$ and $\mathbf{H}$ can be $\alpha \simeq \pm 37^{\circ}$ or $\alpha \simeq \pm 143^{\circ}$.

There can be also a resonance situation when the period of the modulation of the interlayer coupling $\lambda_{H}=2 \phi_{0} / d H$ becomes equal to the period of FFLO modulation $\lambda_{\text {FFLO }}$ (for $\left.T=0 \lambda_{\mathrm{FFLO}}^{0}=\pi \hbar v_{F} / \Delta_{0}=\pi^{2} \xi_{0}\right)[20,21]$. In the reso- nance, the direction of the FFLO modulation vector can be further tilted from its direction in the out of the resonance case. Since the resonance position depends on the strength of the external magnetic field, we can find situations when the direction of the FFLO modulation vector is also field dependent. Figure 4 exhibits the field direction dependence of the normalized superconducting transition temperature, $T_{c}(\alpha) / T_{c P}$ for $\eta=1.75$ and $\eta=2.07$, when the resonance conditions are possible. The direction of the modulation vector is fixed by the cusps in the angle dependence of the onset superconducting temperature.

In this work, we have studied the effect of the applied inplane magnetic field on the orientation of the FFLO modulation vector in $s$-wave paired layered superconductors. We showed that in the vicinity of TCP the orbital effect keeps the direction of the modulation perpendicular to the direction of the field. In the low temperature regime, the FFLO modulation can acquire different direction when the absolute value of the Fermi velocity is changed or when the resonance between the modulation and the field-induced interlayer coupling develops.

Acknowledgements We acknowledge the support by the European Community under a Marie Curie IEF Action (Grant Agreement No. PIEF-GA-2009-235486-ScQSR) and European IRSES program SIMTECH.

\section{References}

1. Larkin, A.I., Ovchinnikov, Yu.N.: Zh. Eksp. Teor. Fiz. 47, 1136 (1964) [Sov. Phys. JETP 20, 762 (1965)]

2. Fulde, P., Ferrell, R.A.: Phys. Rev. 135, A550 (1964)

3. Aslamazov, L.G.: Zh. Eksp. Teor. Fiz. 55, 1477 (1968) [Sov. Phys. JETP 28, 773 (1969)]

4. Singleton, J., et al.: J. Phys. Condens. Matter 12, L641 (2000)

5. Tanatar, M.A., Ishiguro, T., Tanaka, H., Kobayashi, H.: Phys. Rev. B 66, 134503 (2002) 
6. Bianchi, A., Movshovich, R., Oeschler, N., Gegenwart, P., Steglich, F., Thompson, J.D., Pagliuso, P.G., Sarrao, J.L.: Phys. Rev. Lett. 89, 137002 (2002)

7. Miclea, C.F., Nicklas, M., Parker, D., Maki, K., Sarrao, J.L., Thompson, J.D., Sparn, G., Steglich, F.: Phys. Rev. Lett. 96, 117001 (2006)

8. Uji, S., Terashima, T., Nishimura, M., Takahide, Y., Konoike, T., Enomoto, K., Cui, H., Kobayashi, H., Kobayashi, A., Tanaka, H., Tokumoto, M., Choi, E.S., Tokumoto, T., Graf, D., Brooks, J.S.: Phys. Rev. Lett. 97, 157001 (2006)

9. Shinagawa, J., Kurosaki, Y., Zhang, F., Parker, C., Brown, S.E., Jérome, D., Christensen, J.B., Bechgaard, K.: Phys. Rev. Lett. 98, 147002 (2007)

10. Lortz, R., Wang, Y., Demuer, A., Böttger, P.H.M., Bergk, B., Zwicknagl, G., Nakazawa, Y., Wosnitza, J.: Phys. Rev. Lett. 99, 187002 (2007)

11. Cho, K., Smith, B.E., Coniglio, W.A., Winter, L.E., Agosta, C.C., Schlueter, J.A.: Phys. Rev. B 79, 220507(R) (2009)

12. Wright, J.A., Green, E., Kuhns, P., Reyes, A., Brooks, J., Schlueter, J., Kato, R., Yamamoto, H., Kobayashi, M., Brown, S.E.: Phys. Rev. Lett. 107, 087002 (2011)

13. Bergk, B., Demuer, A., Sheikin, I., Wang, Y., Wosnitza, J., Nakazawa, Y., Lortz, R.: Phys. Rev. B 83, 064506 (2011)

14. Coniglio, W.A., Winter, L.E., Cho, K., Agosta, C.C., Fravel, B., Montgomery, L.K.: Phys. Rev. B 83, 224507 (2011)
15. Agosta, C.C., Jin, J., Coniglio, W.A., Smith, B.E., Cho, K., Stroe, I., Martin, C., Tozer, S.W., Murphy, T.P., Palm, E.C., Schlueter, J.A., Kurmoo, M.: Phys. Rev. B 85, 214514 (2012)

16. Uji, S., Kodama, K., Sugii, K., Terashima, T., Takahide, Y., Kurita, N., Tsuchiya, S., Kimata, M., Kobayashi, A., Zhou, B., Kobayashi, H.: Phys. Rev. B 85, 174530 (2012)

17. Yonezawa, S., et al.: Phys. Rev. Lett. 100, 117002 (2008)

18. Croitoru, M.D., Houzet, M., Buzdin, A.I.: Phys. Rev. Lett. 108, 207005 (2012)

19. Croitoru, M.D., Houzet, M., Buzdin, A.I.: J. Supercond. Nov. Magn. 25, 1283 (2012)

20. Croitoru, M.D., Buzdin, A.I.: Phys. Rev. B 86, 064507 (2012)

21. Croitoru, M.D., Buzdin, A.I.: Phys. Rev. B 86, 224508 (2012)

22. Tsuzuki, T.: J. Low Temp. Phys. 9, 525 (1972)

23. Brison, J.P., Keller, N., Vernière, A., Lejay, P., Schmidt, L., Buzdin, A., Flouquet, J., Julian, S.R., Lonzarich, G.G.: Physica C 250, 128 (1995)

24. Denisov, D., Buzdin, A., Shimahara, H.: Phys. Rev. B 79, 064506 (2009)

25. Kopnin, N.B.: Theory of Nonequilibrium Superconductivity. Clarendon, Oxford (2001)

26. Bulaevskii, L.N.: Zh. Eksp. Teor. Fiz. 65, 1278 (1973) [Sov. Phys. JETP 38, 634 (1974)] 